\title{
With thanks to our 2010 peer reviewers
}

We are grateful to the following people for their significant contribution to Chronic Diseases in Canada as peer reviewers in 2010. Their expertise ensures the quality of our journal and promotes the sharing of new knowledge among peers in Canada and internationally.

Devendra Amre

Kelly Anderson

Chris Andrews

Sten Ardal

Megan Aston

George Beaton

Nicholas Birkett

Carole Blanchet

Larry Chambers

Yves Chaput

Yue Chen

Margaret Cheney

Mary Chipman

Wong Ho Chow

Lynda Corby

Simone Dahrouge

Carl D'Arcy

Joseph DiFranza

Roland Dyck

Grace Egeland

Garry Egger

Daniel Fuller

Tracey Galloway

Didier Garriguet

Leslie Gaudette

William Gnam

Katherine Gray-Donald

Judy Guernsey

Leona Hakkaart-Van Roijen

Anne-Marie Hamelin

Trevor Hancock

Lisa Hartling

Ken Hoffman

Janie Houle

Jennifer Hutcheon

Brian Hutchison

K. S. Joseph

Anita Koushik

Yvonne Lamers

Jérôme Lavoué

Nancy Lightfoot

Shiliang Liu

Lisa Lix

Francine Lortie-Monette

Pat Martens
Jane McCusker

Rena Mendelson

Les Mery

Christiana Miewald

Anthony Miller

Judy Morona

John Myers

Bruce Newbold

Jill Newstead-Angel

Edward Ng

Michael Otterstatter

Gordon Phaneuf

Will Pickett

Robert Platt

Kevin Pottie

Sampsa Puttonen

Georgia Roberts

Elizabeth Robinson

Edmond Ryan

Diana Schendel

Cindy Scythes

Monique Séguin

David Streiner

Paul Taenzer

Mary Thompson

Ross Tsuyuki

Ana Velly

Harth Volker

Kitty Wilkins

Kristy Wittmeier

Margareth Zanchetta 